\title{
FUSÃO DE IMAGENS ALTIMÉTRICAS E AEROMAGNETOMÉTRICAS COMO FERRAMENTA DE INTERPRETAÇÃO GEOLÓGICA, EXEMPLO DA PROVÍNCIA MINERAL DE CARAJÁS (PA)
}

\author{
Cleyton de Carvalho Carneiro ${ }^{1}$, Alvaro Penteado Crósta $^{2}$, \\ Adalene Moreira Silva ${ }^{3}$ e Roberto Vizeu Lima Pinheiro ${ }^{4}$ \\ Recebido em 20 março, 2006 / Aceito em 22 junho, 2006 \\ Received on March 20, 2006 / Accepted on June 22, 2006
}

\begin{abstract}
The Carajás Mineral Province is located in the eastern portion of the Amazonian Craton, in the Pará state, north of Brazil. It comprises two main tectonic domains: Carajás and Cinzento strike-slip systems. A significant proportion of the mineral deposits and occurrences of this Province is geologically related to structures belonging to these two fault systems. This paper presents the results obtained by the interpretation of merged altimetric and magnetometric data, as an auxiliary tool for understanding the structural framework of the Carajás Fault. Satellite digital elevation data acquired by the Shuttle Radar Topographic Mission (SRTM) were used in combination with aeromagnetometric data acquired by the Brazil-Canada Geophysical Project (PGBC). A method was developed for digital processing and merging the data acquired by these two surveys, based on geophysical and remote sensing processing methods. The results obtained allowed to establish the relationship between features interpreted from the merged image, with the main geomorphologic and magnetic domains known in the region. These results demonstrate the potential of applying these methods for assessing areas with similar metallogenetic characteristics located elsewhere in Amazon, thus facilitating and guiding regional exploration programs.
\end{abstract}

Keywords: Data fusion, Remote Sensing, Aerogeophysics, Carajás Mineral Province.

RESUMO. A Província Mineral de Carajás localiza-se na parte sudeste do Cráton Amazônico, estado do Pará. É composta por dois domínios tectônicos principais, representados pelos sistemas transcorrentes Carajás e Cinzento. Parte significativa das ocorrências minerais da referida província está vinculada geologicamente a estruturas que compõem esses dois sistemas de falhas. Este trabalho apresenta os resultados obtidos da interpretação realizada a partir da fusão de dados magnetométricos e altimétricos, como ferramenta auxiliar na compreensão do quadro estrutural relacionado à Falha Carajás. Foram utilizadas as imagens orbitais do modelo digital de elevação, gerado a partir dos dados altimétricos orbitais da Shuttle Radar Topographic Mission (SRTM), e dos dados aeromagnetométricos do Projeto Geofííco Brasil-Canadá (PGBC). Para atingir os objetivos propostos foi desenvolvido um método de processamento e fusão dos dados provenientes dos dois levantamentos, geofísico e altimétrico, a partir de técnicas de processamento geofísico e de imagens digitais de sensoriamento remoto. Os resultados obtidos permitiram estabelecer a relação das feições interpretadas a partir da imagem de fusão com os principais domínios geomorfológicos e magnéticos reconhecidos na região. Estes resultados demonstram o potencial de aplicação desses métodos na análise de outras áreas com características metalogenéticas similares às da Amazônia, como forma de facilitar e orientar programas regionais de exploração mineral.

Palavras-chave: Fusão de imagens, Sensoriamento Remoto, Aerogeofísica, Província Mineral de Carajás.

\footnotetext{
${ }^{1}$ Instituto de Geociências, Universidade Estadual de Campinas, Caixa Postal 6152 - Campinas, SP. Tel: (19) 3521-4599; Fax: (19) 3289-6215 - E-mail: cleyton@ige.unicamp.br

2 Instituto de Geociências, Universidade Estadual de Campinas, Caixa Postal 6152 - Campinas, SP. Tel: (19) 3521-5120; Fax: (19) 3289-6215 - E-mail: alvaro@ige.unicamp.br

3 Instituto de Geociências, Universidade de Brasília, Campus Universitário Darcy Ribeiro, Asa Norte, Brasília, DF. Tel: (61) 3307-2877; Fax: (61) 3347-4062

-E-mail: adalene@unb.br

${ }^{4}$ Centro de Geociências, Universidade Federal do Pará, Caixa Postal 8608 - Belém, PA. Tel: (91) 3201-7393; Fax: (91) 3201-7609 - E-mail: vizeu@ufpa.br
} 


\section{INTRODUÇÃo}

Imagens produzidas a partir de dados aerogeofísicos e de sensores remotos são utilizadas rotineiramente como ferramentas de mapeamento geológico, embora na maioria das vezes de forma não integrada. Por outro lado, quando estes mesmos tipos de dados são utilizados de forma integrada, por meio de fusões de imagens utilizando técnicas de processamento digital, informações litológicas e estruturais que muitas vezes são imperceptíveis nas imagens individuais tornam-se mais evidentes à visão humana. Conseqüentemente, imagens geradas por fusão de dados multifontes podem auxiliar sobremaneira o mapeamento geológico tradicional, sendo cada vez mais utilizadas (Henderson \& Lewis, 1998; Rencz, 1999; Drury, 2001).

No Brasil, os dados comumente utilizados em fusão de imagens são a gamaespectrometria e o sensoriamento remoto, tanto no espectro ótico como no de microondas (Dias \& Paradella, 1997; Paradella et al., 1997; Paradella et al., 2000; Santos et al., 1997; Teruiya, 2002; Madrucci et al., 2003).

Fusões entre imagens de sensoriamento remoto e aeromagnetométricas, entretanto, são mais raras, encontrando-se poucos exemplos na literatura (Teixeira, 2003; Teixeira et al., 2004; Carneiro, 2005). 0 uso combinado desses dois tipos de dados requer certo cuidado metodológico e interpretativo, principalmente por reunir informações de diferentes níveis crustais e de naturezas bastante distintas. No caso do sensoriamento remoto no espectro ótico, as imagens produzidas expressam a resposta espectral relacionada à composição química/mineralógica dos materiais superficiais. Já no sensoriamento remoto no espectro das microondas, utilizando sensores do tipo radar (SAR - synthetic aperture radar), as informaç̧ões texturais das imagens são relacionadas às características físicas do terreno, ao relevo superficial e às propriedades elétricas dos materiais. Já as imagens aeromagnetométricas registram o gradiente magnético em níveis distintos de profundidade. Além disso, o campo magnético em regiões de baixas latitudes é representado por anomalias dipolares, e o posicionamento dos dipolos magnéticos nestas regiões não coincide com a respectiva fonte magnética geradora da anomalia. Neste contexto, para a observação da anomalia magnética sobre sua respectiva fonte causadora, torna-se necessária a aplicação de filtros tais como redução ao pólo, redução ao equador ou amplitude do sinal analítico (Luiz \& Silva, 1995; Blum, 1999; Silva, 1999; Nabighian et al., 2005).

A Província Mineral de Carajás, localizada na região sudeste do estado do Pará, nos domínios da floresta amazônica, compreende abundantes e diversificados depósitos minerais. Contudo, as atividades exploratórias são dificultadas pela impossibilidade de acesso direto a afloramentos de rochas, para a coleta de dados e caracterização de alvos potenciais para mineralização. Isto se deve à presença da densa cobertura vegetal, principalmente em áreas de reservas florestais, e do espesso manto intempérico típicos da Amazônia, fazendo com que métodos exploratórios tradicionais nem sempre tenham o êxito desejado.

Muitos dos depósitos minerais dessa região mostram relações com variações no gradiente magnético e também com estruturas geológicas como falhas dilatacionais. Estas últimas expressam-se como feições geomorfológicas (Carneiro, 2005), sendo evidenciadas em imagens obtidas a partir de modelos digitais de elevação (MDE).

Este trabalho analisa a fusão de imagens MDE e aeromagnetométricas na Província Mineral de Carajás, como forma de otimizar 0 emprego de dados indiretos em programas exploratórios na Amazônia, buscando minimizar os obstáculos acima mencionados. Para tanto, foram utilizadas imagens altimétricas geradas pela Shuttle Radar Topographic Mission (SRTM), a partir da tecnologia de interferometria por radar (InSAR), e imagens magnetométricas geradas a partir dos dados do Projeto Aerogeofísico Brasil-Canadá (PGBC), integradas por meio de técnicas de fusão digital.

\section{CONTEXTO GEOLÓGICO REGIONAL}

A Província Mineral de Carajás está localizada na porção leste do Escudo Brasil Central, nos domínios da Província MaroniItacaiúnas (Tassinari \& Macambira, 2004). É subdividida em duas unidades geotectônicas principais:

(1) 0 Cinturão Itacaiúnas (Araújo et al., 1988; Araújo \& Maia, 1991), a norte; $e$

(2) o Terreno Granito-Greenstone Rio Maria (Dall'Agnol et al., 1987 e 1997; Althoff, 1996), a sul.

Essas unidades estão bordejadas a leste pelo Cinturão Araguaia e a oeste pela Província Proterozóica do Sul do Pará. A borda norte da província está coberta, de forma transicional, por rochas supracrustais do domínio tectônico Bacajá.

0 Cinturão Itacaiúnas, onde está localizada a área de trabaIho, apresenta como subdomínios tectônicos principais os sistemas transcorrentes Carajás e Cinzento (Fig. 1). A Falha Carajás representa a principal estrutura do Sistema Transcorrente de mesmo nome. É composta por lineamentos descontínuos, anastomóticos, cuja direção preferencial é NW-SE. Apresenta uma extensão total de aproximadamente $180 \mathrm{~km}$ e terminações em splays (Pinheiro, 1997). 

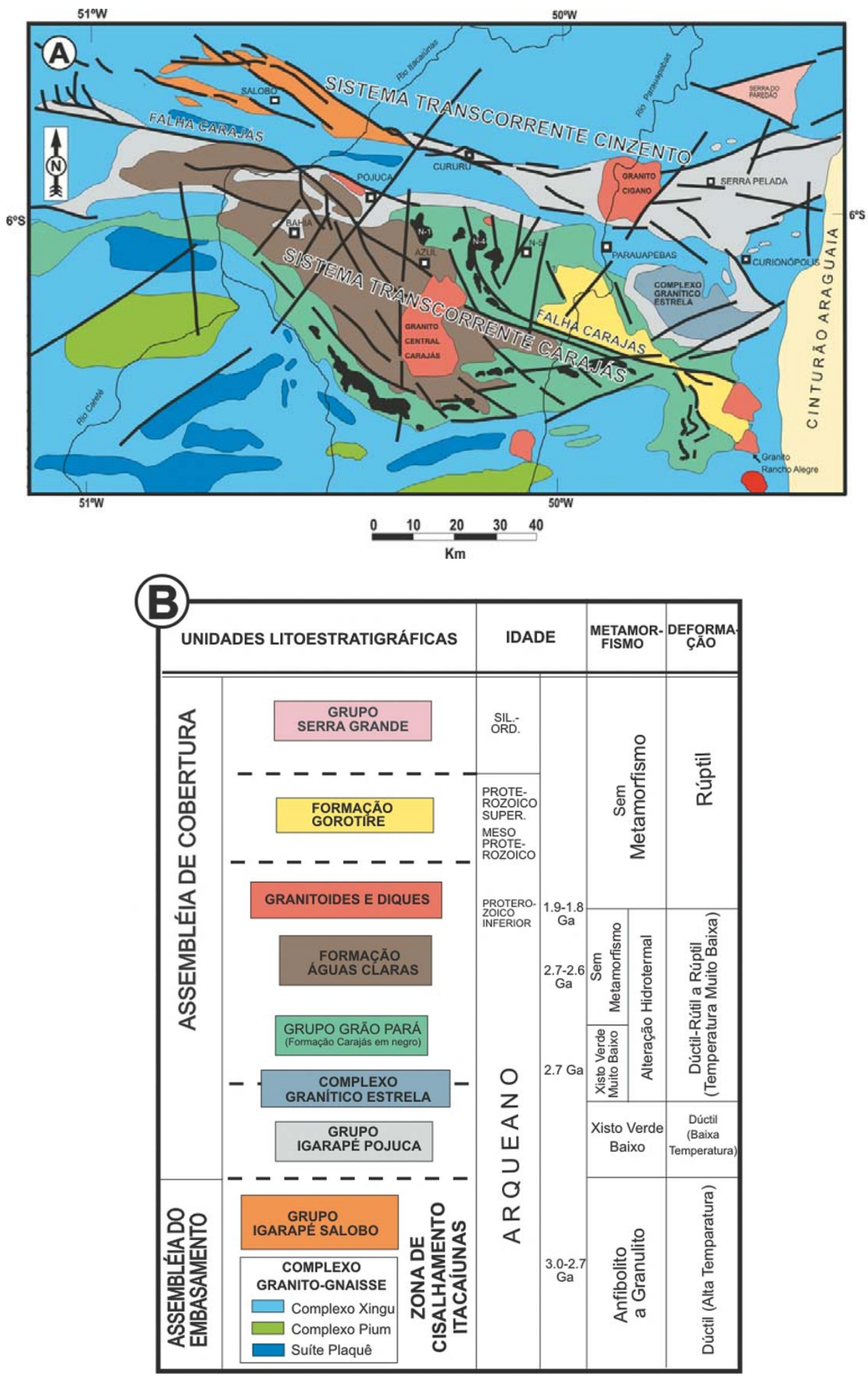

Figura 1 - (A) Mapa geológico simplificado do Cinturão Itacaiúnas evidenciando os sistemas transcorrentes Carajás e Cinzento; (B) Quadro tectonoestratigráfico de Carajás (Pinheiro, 1997). 
Muitas tentativas de organizações estratigráficas e tectônicas foram propostas para a região de Carajás (DOCEGEO, 1988; Araújo \& Maia, 1991; Pinheiro, 1997; Veneziani et al., 2004). A proposta de Pinheiro (1997) obedece a critérios tectonoestratigráficos, subdividindo as rochas da região em:

(1) Assembléia do Embasamento; e

(2) Assembléia de Cobertura.

\section{Assembléia do Embasamento}

\section{Complexo Granito-Gnáissico}

É formado pelos conjuntos litológicos arqueanos do Complexo Pium (DOCEGEO 1988), Complexo Xingu (Silva et al., 1974), granitóides deformados e metamorfisados da Suíte Plaquê.

0 Complexo Pium (DOCEGEO, 1988) é composto por granulitos félsicos e máficos (Araújo \& Maia, 1991) que afloram principalmente na porção sul da Serra dos Carajás. Em datações SHRIMP U-Pb, Pidgeon et al. (2000) obtiveram uma idade de 3.002 土 14 Ma para a cristalização do protólito e $2.859 \pm 9 \mathrm{Ma}$ para 0 evento metamórfico de fácies granulito que afetou as rochas desse complexo.

0 Complexo Xingu (Silva et al., 1974) corresponde a um conjunto de rochas gnáissicas tonalíticas, trondjemíticas, granodioríticas, graníticas e anfibolíticas (Araújo \& Maia, 1991). Através do método U/Pb em zircão, Machado et al. (1991) obtiveram uma idade de $2.859 \pm 2$ Ma para o episódio de migmatização dessas rochas.

Nos últimos anos foi evidenciada a ocorrência de um evento de granitogênese regional datado entre 2,73 - 2,76 Ga (Avelar et al., 1994; Huhn et al., 1999; Barros et al., 2001; Barbosa et al., 2005), responsável pela intrusão de diversos corpos graníticos nos domínios antes atribuídos ao Complexo Xingu, tais como Complexo Granítico Estrela, Granito Planalto, Granito Rancho Alegre, Suíte Plaquê e Rochas Graníticas do Igarapé Gelado no domínio das rochas do embasamento e supracrustais.

\section{Assembléia de Cobertura}

São representadas por sequiências vulcano-sedimentares arqueanas (Pinheiro, 1997). 0 autor reagrupou as rochas do até então denominado Supergrupo Itacaiúnas (DOCEGE0, 1988) como pertencentes a um único conjunto, eventualmente diferenciado por localização, intensidade de deformação e mesmo intensidade de alteração hidrotermal/metamórfica. Nesta proposta, permaneceram os Grupos Igarapé Pojuca e Grão Pará. Suas datações apontam para um intervalo curto situado em torno de 2,7 Ga.
As rochas das formações Águas Claras (Nogueira et al., 1995) e Gorotire (Lima \& Pinheiro, 2001) representam as seqüências sedimentares clásticas da região.

Os plútons e diques de granitos anorogênicos paleoproterozóicos ocorrem na forma de diversas intrusões nas rochas do embasamento e supracrustais. Dentre eles, estão 0 Granito Cigano e 0 Granito Central de Carajás ambos datados em 1,88 Ga (Machado et al., 1991).

\section{PROCESSAMENTO DO MODELO DIGITAL DE ELEVAÇÃO SRTM/INSAR}

Os dados do modelo digital de elevação foram gerados pela "Shuttle Radar Topography Mission" (SRTM). A missão utilizou a tecnologia de interferometria SAR (InSAR) de passagem única, composta por duas antenas de radar (SAR) separadas 60 metros entre si, por meio de uma haste projetada para fora do corpo do ônibus espacial. Esses dados foram coletados entre os dias 11 e 22/02/2000 pelo ônibus espacial Endeavour e proporcionaram a geração de dados altimétricos cobrindo cerca de $80 \%$ da superfície da Terra. Utilizados na forma de modelo digital de elevação (MDE), esses dados altimétricos têm resolução radiométrica de 16-bit (intervalo de 65.535 valores possíveis para representar a variável altitude) e resolução espacial original de 1 arc sec ( $30 \mathrm{~m})$. Os dados disponibilizados pela NASA para a América do Sul, assim como para todas as demais regiões da Terra exceto a América do Norte, foram submetidos à reamostragem espacial para 3 arc sec $(\sim 90 \mathrm{~m})$. A SRTM operou simultaneamente com duas antenas, respectivamente nas bandas C e X (Rabus et al., 2003).

Na composição do modelo digital de elevação de Carajás foram utilizados os dados da banda $\mathrm{C}$ com resolução espacial de 3 arc sec, disponibilizados pela National Aeronautics and Space Administration (NASA) dos Estados Unidos.

0 mosaico, que compreende toda a Província de Carajás e adjacências, foi elaborado a partir de 15 quadrículas de $1 \times 1$ grau entre as coordenadas 53W/4S e 48W/7S. Com este mosaico, selecionou-se a área do Cinturão Itacaiúnas para o processamento e posterior interpretação geológica. Técnicas de colorização artificial (pseudo-cor ou fatiamento de cores) foram utilizadas para representar intervalos altimétricos do MDE em diferentes cores, de forma a realçar diferenças topográficas mais sutis. Em seguida, foram aplicados: filtros de convolução do tipo mediana adaptativa, com dimensão $3 \times 3$ elementos, para suavização e remoção de imperfeições; filtros passa-altas de iluminação direcional artificial, com variação interativa dos 
ângulos de azimute e elevação da iluminação; e, por fim, ajuste linear de histogramas para otimização do intervalo dinâmico de valores do MDE (Crósta, 1992). Esse conjunto de técnicas teve por finalidade o realce dos atributos foto-interpretativos de interesse presentes nos dados, tendo em vista o reconhecimento de feições morfológicas na imagem da superfície.

\section{PROCESSAMENTO DOS DADOS AEROMAGNETO- MÉTRICOS}

Os dados aeromagnetométricos foram gerados pelo convênio entre 0 Departamento Nacional de Pesquisa Mineral (DNPM) do Ministério das Minas e Energia (MME) e a Agência Canadense para o Desenvolvimento Internacional (Canadian International Development Agency - CIDA). Estes dados fazem parte do Projeto Geofísico Brasil-Canadá (PGBC-1020), que compõe um dos 63 projetos da Série 1000 realizada entre os anos de 1953 e 2001 . A amostragem dos dados referentes ao PGBC-1020 que inclui a região de Carajás foi feita nos anos de 1975 e 1976 (DNPM, 1981).

A área total do PGBC-1020 cobre parte dos estados de Goiás, Tocantins, Mato Grosso, Pará e Maranhão (Fig. 2). No projeto, subdividido em três blocos, foram levantados dados magnéticos e gamaespectrométricos em uma área de $375.000 \mathrm{~km}^{2}$, com intervalos de amostragem de 1 segundo ao longo dos perfis. As linhas de produção, orientadas na direção N-S, apresentam espaçamento de $2 \mathrm{~km}$ no levantamento de reconhecimento. Posteriormente, em um levantamento complementar de semidetalhe, houve 0 adensamento das linhas de produção dos blocos para 0 espaçamento de $1 \mathrm{~km}$. 0 sobrevôo das linhas de controle foi realizado na direção E-W, com espaçamento entre linhas de $14 \mathrm{~km}$. As medidas de intensidade do campo magnético foram obtidas a partir de magnetômetro Fluxgate e registradas em intervalos de 70 m, com precisão de $1 \mathrm{nT}$.

Os dados aeromagnetométricos foram submetidos às etapas de processamento descritas a seguir.

\section{Pré-processamento}

A primeira etapa consistiu no controle adicional de qualidade relacionada às informações que seriam efetivamente usadas. Dessa forma, a análise buscou imperfeições que pudessem prejudicar etapas seguintes, tais como ruídos nas gravações digitais, em todos os registros lógicos que representam a amostragem total. Para isso foram utilizados os métodos denominados de Diferença Quarta e Parâmetro P propostos por Blum (1999), que permitem a identificação de registros cujos valores não se enquadram no desvio-padrão da população de registros, ou seja, valores espúrios ao conjunto de dados. Os dados magnéticos utilizados neste estudo demonstraram a presença de ruídos os quais, após identificados, foram eliminados das linhas de vôo.

\section{Processamento}

0 processamento consistiu em uma série de etapas, ilustradas na Fig. 3. Na geração do campo magnético anômalo houve a remoção da contribuição referente ao campo magnético principal utilizando-se do cálculo matemático apropriado, representado pelo International/Definitive Geomagnetic Reference Field (IGRF/DGRF).

A partir da interpolação do campo magnético anômalo foi gerada uma malha com células de $500 \mathrm{~m}$, correspondentes a $1 / 4$ do espaçamento entre as linhas de produção do projeto, denominada de malha $\mathrm{A}$.

A etapa seguinte consistiu na aplicação da técnica de micronivelamento, para correção de imperfeições notadas no nivelamento da malha A. Estas imperfeições na representação espacial dos dados se devem ao nivelamento convencional dos dados magnéticos, usando linhas de vôo transversais às linhas de produção. Desta forma, houve necessidade de homogeneizar a representação espacial do campo anômalo. Para isso, fez-se uso da técnica de micronivelamento (Minty, 1991). 0 procedimento é implementado por meio das seguintes etapas: (i) aplicação de filtro passa-alta, com comprimento de onda no mínimo igual ao dobro do espaçamento entre as linhas de produção, na direção perpendicular às linhas de produção, resultando em uma malha B; (ii) aplicação de filtro passa-baixa à malha $\mathrm{B}$, com comprimento de onda no mínimo igual ao dobro do espaçamento entre as linhas de controle, na direção da linha de produção, originando uma malha $\mathrm{C}$; (iii) subtração dos resultados da malha $\mathrm{C}$ daqueles da malha A, obtendo a malha final micronivelada.

Após as correções, os dados magnetométricos foram submetidos às transformações necessárias para geração de temas interpretativos. Na análise dos dados aeromagnetométricos foram utilizadas técnicas para a determinação de parâmetros geométricos, tais como a localização de limites geológicos e feições estruturais. Estas técnicas abrangeram 0 cálculo das derivadas vertical e horizontais, bem como a amplitude do sinal analítico. Com isso objetivou-se a demarcação de padrões que individualizassem zonas com comportamento distinto e assinaturas que pudessem traduzir feições magnéticas, isolando-se principalmente aquelas com padrão linear. 


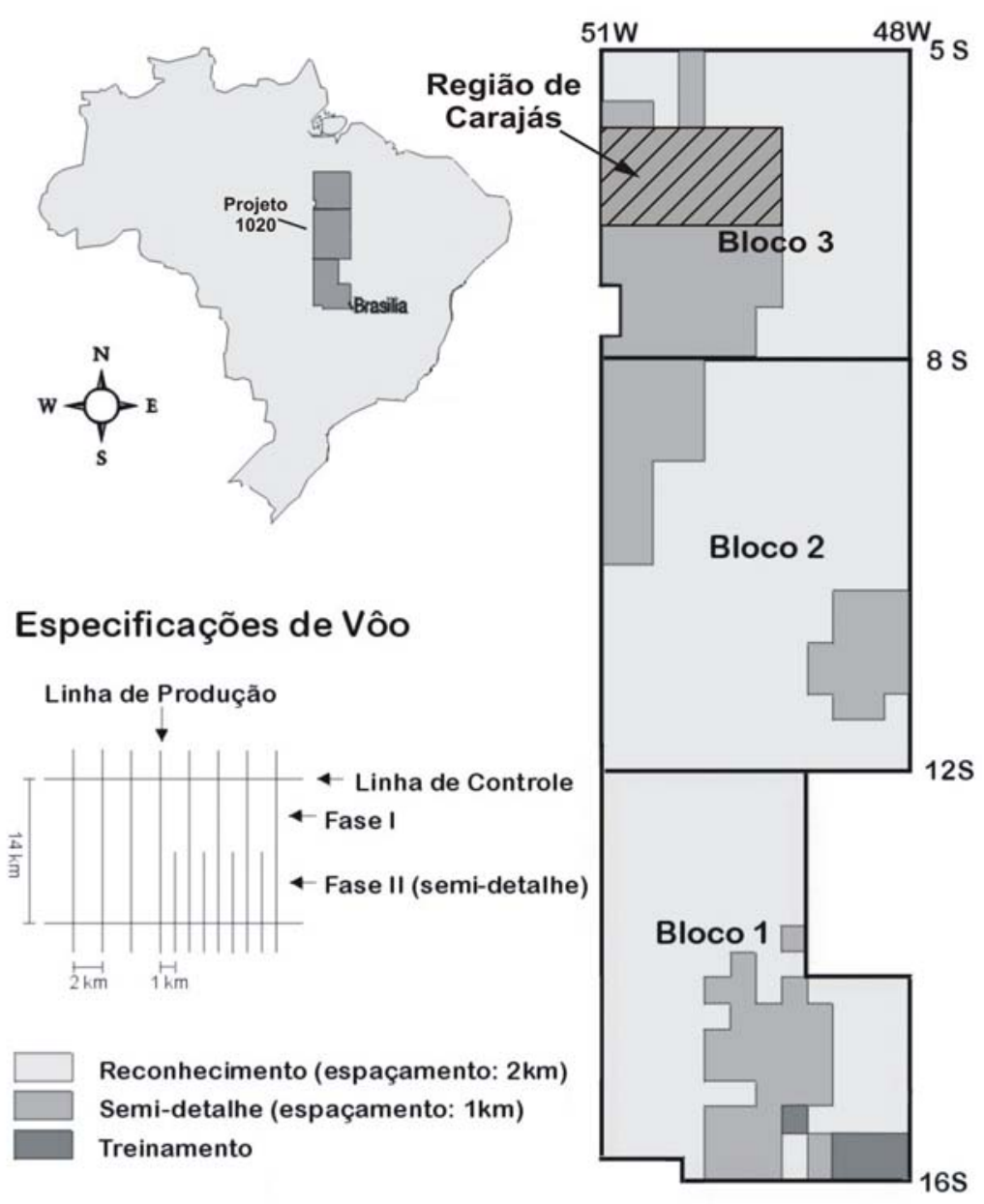

Figura 2 - Projeto aerogeofísicos PGBC - 1020, com destaque para a região de Carajás no Bloco 3. Esta região compreende a área estudada. (Modificado de Blum 1999).

\section{FUSÃO NO DOMÍNIO DO ESPAÇO}

Schowengerdt (1997) descreve a fusão de imagens no domínio espacial como uma técnica voltada para a transferência das informações de alta freqüência, contidas nas imagens de maior resolução espacial, para aquelas de menor resolução espacial, porém com maior resolução no domínio espectral. Este método foi aqui aplicado para a fusão do modelo digital de elevação, representando a imagem com maior resolução espacial $(90 \mathrm{~m})$, com os dados aeromagnetométricos/amplitude do sinal analítico, representando os dados de menor resolução espacial $(500 \mathrm{~m})$. Essa fusão permitiu a análise da variação espacial dos valores de amplitude do sinal analítico, representadas por diferentes tonalidades de cor produzidas pela técnica de fatiamento ou pseudo-cor, ao mesmo tempo em que introduziu os elementos texturais do relevo superficial relativos ao MDE. Além de integrar dados de natureza distinta, a técnica possibilita ainda a análise integrada de dados referentes a diferentes níveis crustais (sub-superfície e superfície).

A fusão dos dados altimétrico e magnetométrico na região de Carajás foi então feita a partir das imagens do relevo sombreado do MDE e da amplitude do sinal analítico do campo magnético anômalo micronivelado, segundo o fluxograma apresentado na Figura 3.

\section{INTERPRETAÇÃO E DISCUSSÃO}

Com base na imagem produzida pela fusão dos dados altimétricos e aeromagnetométricos foram identificados os principais lineamentos com expressão regional na área da Província Mineral de Carajás (Fig. 4). Esses lineamentos são expressos principalmente por cristas (altos alinhados) magnéticas, mudanças abruptas no 
AEROMAGNETOMETRIA
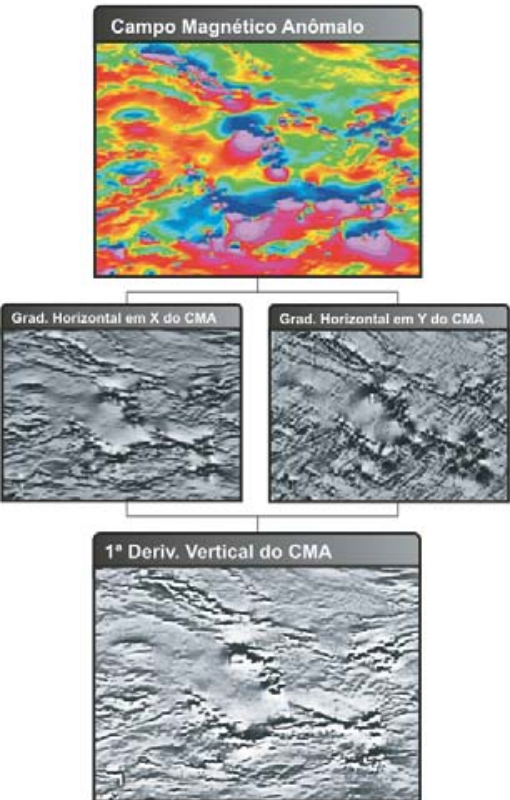

Amplit do Sinal Analitico-ASA
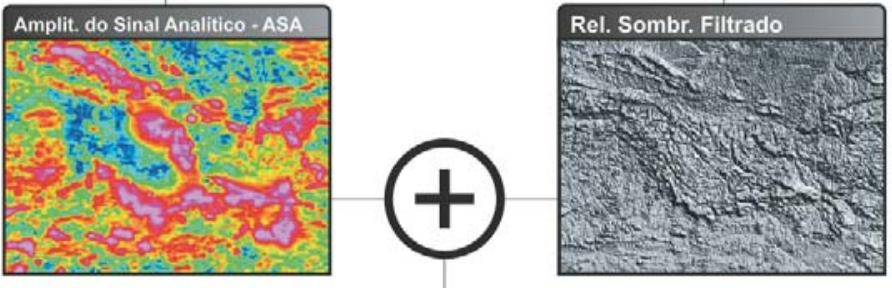

Fusão DEM + ASA

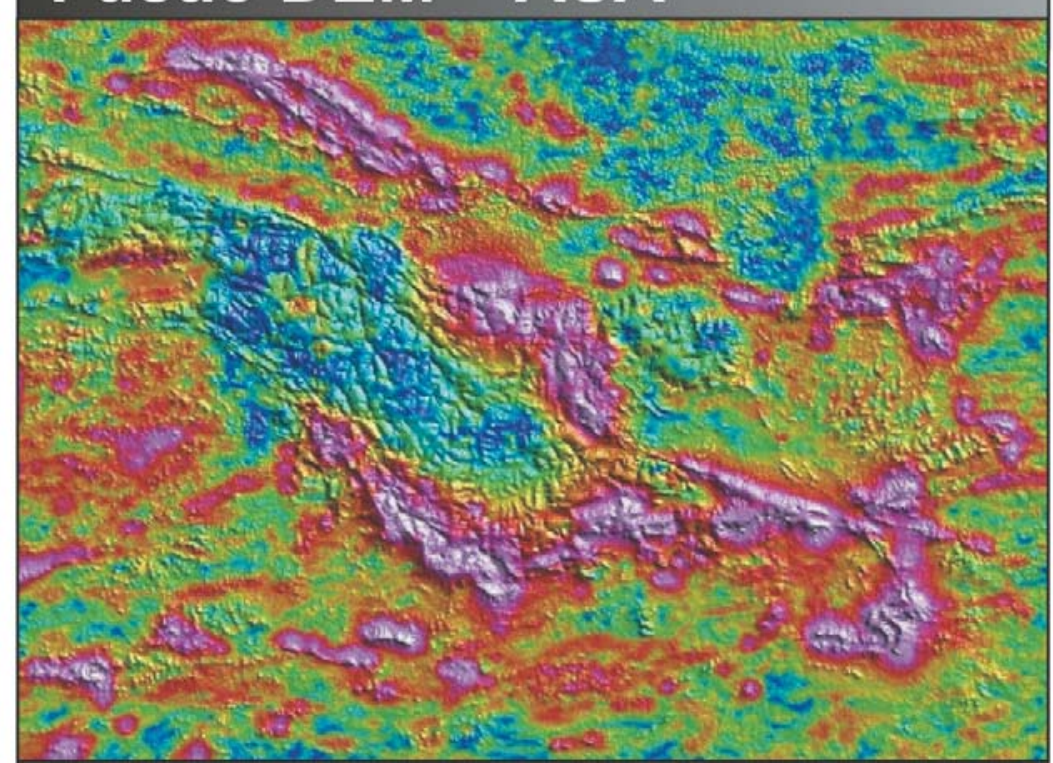

Figura 3 - Fluxograma mostrando as etapas de processamento digital das imagens altimétricas, magnetométricos e fusão entre as imagens.

DEM - SRTM
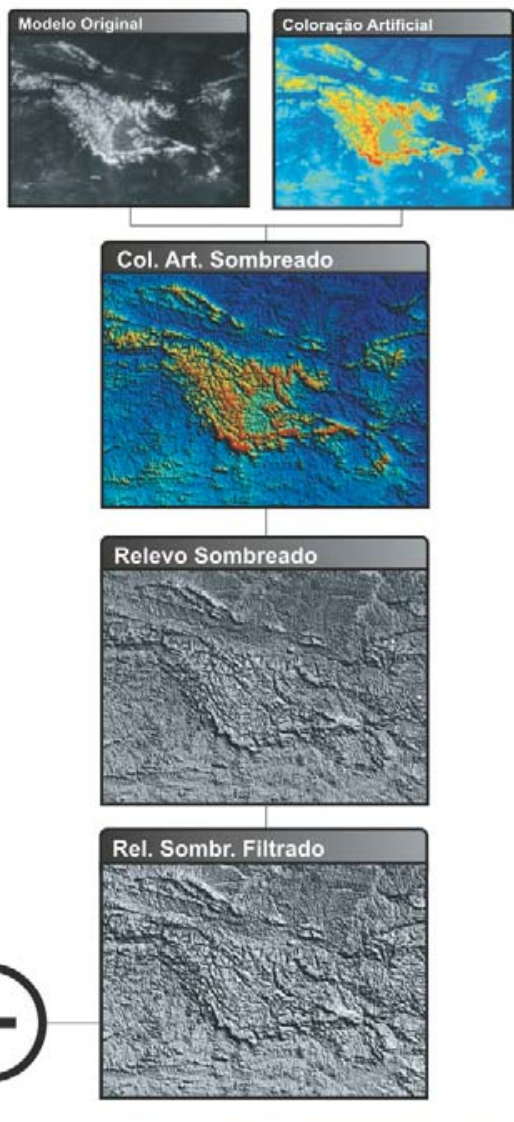


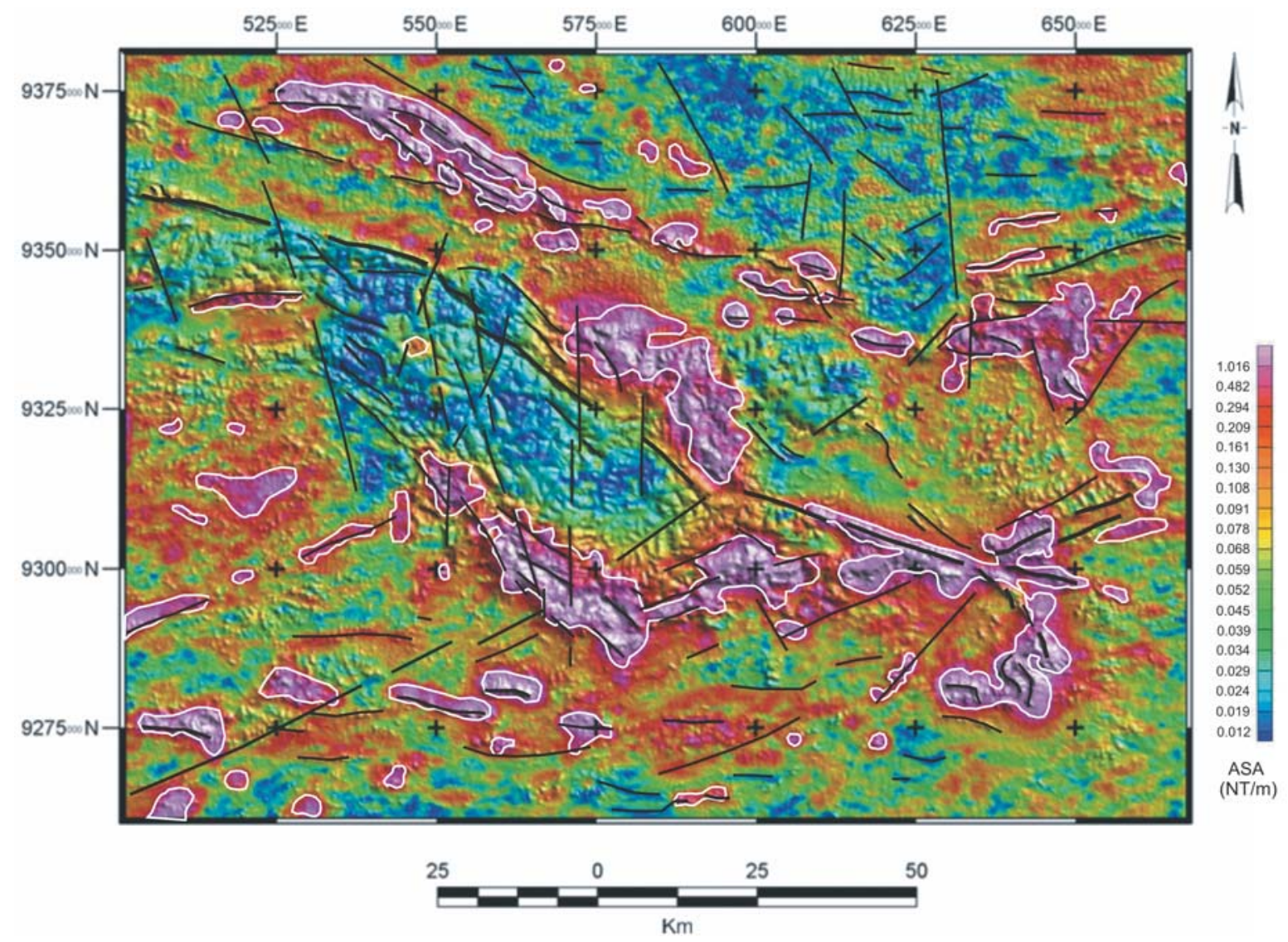

Figura 4 - Imagem ilustrando a fusão dos dados altimétricos e magnetométricos da Província Mineral de Carajás.

gradiente magnético e no relevo superficial.

Pela análise conjunta desses lineamentos, foi possivel observar que: (1) lineamentos de direção preferencial E-W são mais expressivos nos setores norte e sul da área, aparecendo em faixas longas e sinuosas de até $20 \mathrm{~km}$ de extensão que sugerem a presença de possíveis falhas; (2) principalmente no setor central da área ocorrem lineamentos de direção NW-SE, expressos por segmentos retilíneos que formam sigmóides, sobrepostos aos lineamentos E-W anteriormente citados; (3) lineamentos de direção NNW-SSE formam feixes espaçados que deslocam os lineamentos E-W e NW-SE e coincidem com limites marcados por variações abruptas no campo magnético; (4) lineamentos com direções NE-SW e N-S, respectivamente, deslocam os lineamentos de todas as demais direções citadas anteriormente.

Baseado na interpretação da imagem da fusão e dos padrões de lineamentos com características acima descritas, foi possível estabelecer a correlação dos dados interpretados com os principais domínios geomorfológicos reconhecidos na região. As anomalias associadas ao Sistema Transcorrente Cinzento estão re- presentadas por segmentos retilíneos de relevo, que se propagam na direção NW-SE no setor norte da área. 0 setor relacionado à região da Serra Pelada apresenta anomalias evidenciadas por segmentos descontínuos ou não, ora retilíneos ora sinuosos. Setores relacionados às serras Norte e Sul e Serra do Rabo apresentam anomalias magnéticas alongadas e retilíneas.

As zonas individualizadas a partir da imagem da fusão refletem domínios litológicos, os quais foram relacionados a domínios tectônicos e unidades lito-estratigráficas regionais (Fig. 5). Características magnéticas representadas na parte central foram relacionadas às formações ferríferas bandadas do Grupo Grão-Pará. Já as regiões anômalas representadas na parte norte estão relacionadas às rochas do Sistema Transcorrente Cinzento.

\section{CONCLUSÕES}

Filtros como amplitude do sinal analítico demonstram eficácia na determinação de anomalias magnéticas em regiões de baixas latitudes, como é o caso da Província Mineral de Carajás. Pela análise dos elementos obtidos a partir da fusão de da- 


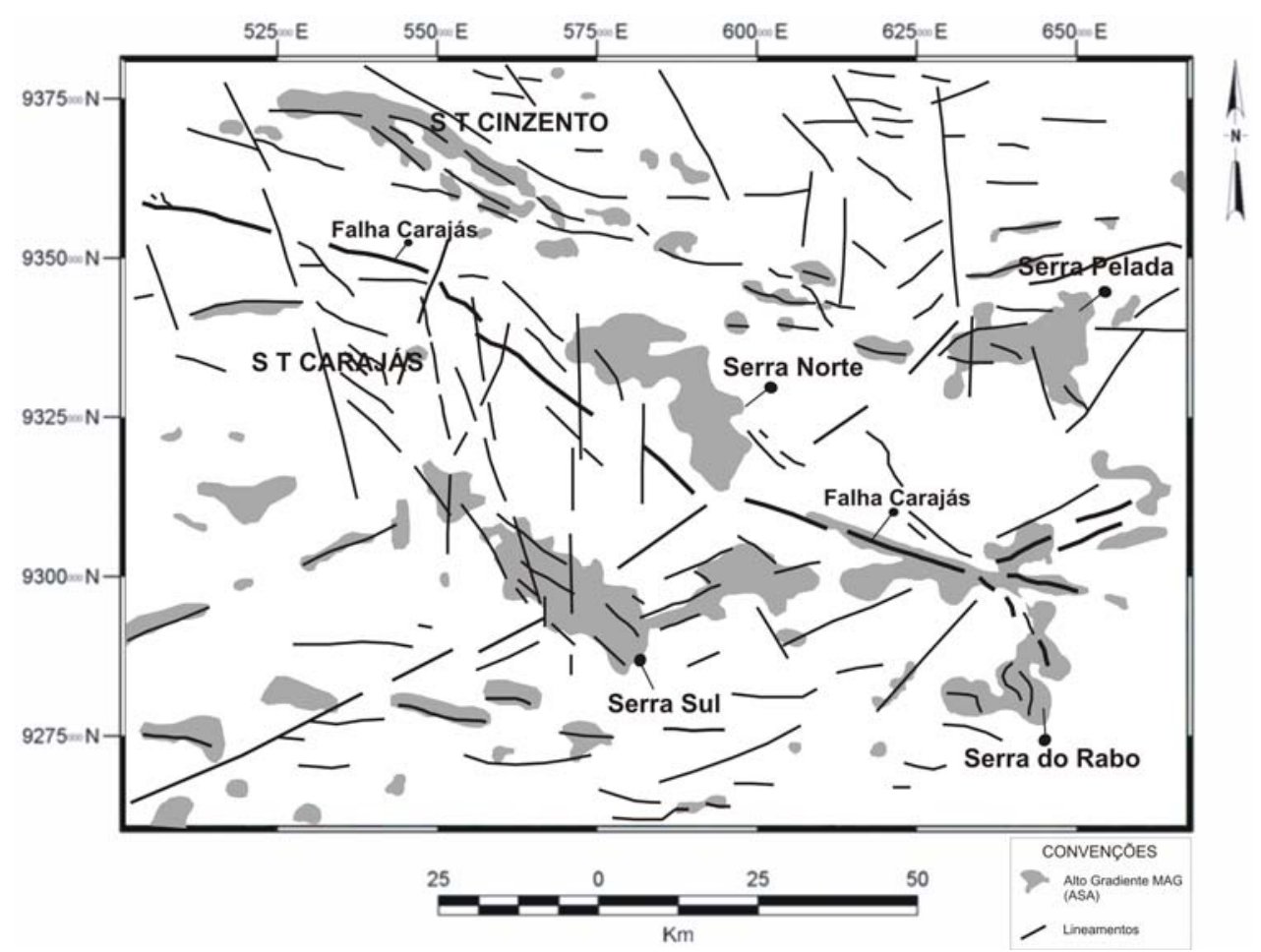

Figura 5 - Mapa de com zonas homólogas de alto gradiente magnético (amplitude do sinal analítico) e lineamentos interpretados pela fusão ASA + DEM.

dos altimétricos e magnéticos nota-se que os sistemas transcorrentes Carajás e Cinzento apresentam domínios magnéticos cujos limites mostram-se coincidentes com as feições geomorfológicas, denotando assim uma correlação entre feições litológicas/estruturais e a formação do relevo.

Variações na composição litológica, assim como mudanças abruptas do gradiente magnético, geradas por unidades geológicas distintas, foram detectadas a partir da imagem de fusão da Província Mineral de Carajás.

Os resultados obtidos neste estudo mostram que a análise integrada de imagens de fusão dos dados altimétricos e magnetométricos pode auxiliar no mapeamento geológico, uma vez que realça variações composicionais, litológicas e estruturais que refletem o substrato geológico, mesmo em regiões de densa cobertura vegetal e espesso manto de solos. Essa abordagem representa uma alternativa eficaz e de baixo custo nas fases iniciais de programas de exploração mineral de abrangência regional, possibilitando 0 aproveitamento de dados aerogeofísicos de baixa resolução, adquiridos com tecnologia dos anos 70 e 80.0 seu emprego na Província Mineral de Carajás destaca as possibilidades interpretativas que a técnica permite, indicando assim seu alto potencial de aplicação em outras áreas da Amazônia.

\section{AGRADECIMENTOS}

Os autores agradecem à FAPESP pela concessão da bolsa de mestrado ao primeiro autor (Proc. No. 02/11984-7); ao Serviço Geológico do Brasil - CPRM, nas pessoas de Luís F. Mourão e Maria Laura V. de Azevedo, pela cessão dos dados aerogeofísicos; e à Companhia Vale do Rio Doce, nas pessoas de Anselmo Soares e José Lancaster, pelo apoio nos trabalhos de campo.

\section{REFERÊNCIAS}

ALTHOFF FJ. 1996. Estude pétrologique et síructurale dês granitóides de Marajoara (Pará, Brésil):leur role dans I 'évolution archéenne du craton Amazonien (2,7-3,2 Ga). Ph.D. Thesis, Nanci, France. Université Henri Poincaré, $296 p$.

ARAÚJO OJB de \& MAIA RGN. 1991. Projeto Especial Mapas de Recursos Minerais, de Solos e de Vegetação para a Área do Programa Grande Carajás; Subprograma Mapas Metalogenéticos e de Previsão de Recursos Minerais; Folha SB.22-Z-A Serra dos Carajás - Estado do Pará. DNPM/CPRM. Brasília, $136 \mathrm{p}$.

ARAÚJO OJB de, MAIA RGN, JORGE JOÃO X da S \& COSTA JBS. 1988. A megaestruturação arqueana da Folha Serra dos Carajás. In: VII Congresso Latino-Americano de Geologia - Anais. Belém, SBG-NO, 1: 324338. 
AVELAR VG, LAFON JM, SCHELLER T, ARAÚJO OJB \& MACAMBIRA EMB. 1994. Geocronologia Pb-Pb por evaporação de zircão e Rb/Sr em rocha total do Granito Seringa, Província Mineral de Carajás. In: XXXVIII Congresso Brasileiro de Geologia, Boletim de resumos expandidos. Balneário de Camboriú, SBG, 2: 387-389.

BARBOSA JPO, BARROS CEM \& MACAMBIRA MJB. 2005. Geocronologia $\mathrm{Pb}-\mathrm{Pb}$ em zircão (evaporação) dos granitos sintectônicos da região do Igarapé Gelado, Província Mineral de Carajás. In: X Simpósio Nacional de Estudos Tectônicos - Anais. Curitiba, SBG-PR, p. 385-387.

BARROS CEM, BARBEY P \& BOULLIER AM. 2001. Role of Magma pressure, tectonic stress and crystallization progress in the emplacement of syntectonic granites. The A-Type Estrela Granite Complex (Carajás Mineral Province, Brazil). Tectonophysics. 343: 93-109.

BLUM MLB. 1999. Processamento e Interpretação de Dados de Geofísica Aérea no Brasil Central e sua Aplicação à Geologia Regional e à Prospecção Mineral. Tese de Doutorado, Instituto de Geociências, Universidade de Brasília, $229 p$.

CARNEIRO CC. 2005. Interpretação de Dados de Sensores Remotos e Aerogeofísicos como Ferramenta na Análise Estrutural da Área CentroOeste do Sistema Transcorrente Carajás (PA). Dissertação de Mestrado, Instituto de Geociências, Universidade Estadual de Campinas, 86 p.

CRÓSTA AP. 1992. Processamento Digital de Imagens de Sensoriamento Remoto. Instituto de Geociências, UNICAMP. Campinas, 164 p.

DALL'AGNOL R, BITTENCOURT JS, JOÃO XSJ, MEDEIROS H, COSTI HT \& MACAMBIRA MJB. 1987. Granitogenesis in northern Brazilian region: a review. Revista Brasileira de Geociências, 17: 382-403.

DALL'AGNOL R, SOUZA ZS, ALTHOFF FJ, BARROS CEM, LEITE AAS \& JORGE JOÃO XS. 1997. General aspects of the granitogenesis of the Carajás metallogenic province. In: II International Symposium on Granite and Associated Mineralizations, Extended abstract sand program. Salvador, p. 135-161.

DIAS RR \& PARADELLA WR. 1997. Integração de dados aéreos gamaespectrométricos com imagens TM-Landsat no mapeamento geológico da área do Pojuca, Província Mineral de Carajás. Revista Brasileira de Geofísica, 15: 23-33.

DNPM. Departamento Nacional de Produção Mineral. 1981. Projeto Geofísico Brasil-Canadá: histórico e atividades até 30/09/77. MME/DNPM, Goiânia, GSC, Canadá.

DOCEGEO. Rio Doce Geologia e Mineração S.A. 1988. Revisão Litoestratigráfica da Província Mineral de Carajás. In: XXXV Congresso Brasileiro de Geologia, anexo, Anais. Belém, SBG, p. 10-54.

DRURY SA. 2001. Image Interpretation in Geology. $3^{\text {rd }}$ edition. Malden, MA. Blackwell Science. 296 pp.

HENDERSON FM \& LEWIS AJ. 1998. Principles and Applications of Imaging Radar. In: Manual of Remote Sensing, $3^{\text {rd }}$ edition, volume 2.
John Wiley/American Society for Photogrammetry and Remote Sensing. $866 \mathrm{pp}$.

HUHN SRB, SOUZA CIJ, ALBUQUERQUE MC, LEAL ED \& BRUSTOLIN V. 1999. Descoberta do depósito Cu(Au) Cristalino: geologia e mineralização associada - Região da Serra do Rabo - Carajás - PA. In: SBG/Núcleo Norte, Simpósio de Geologia da Amazônia, 6, Manaus, Boletim, 140-143.

LIMA FD \& PINHEIRO RVL. 2001. As Rochas Sedimentares Clásticas (Formação Gorotire) da Terminação Leste da Falha Carajás, Serra dos Carajás - PA. In: REIS NJ \& MONTEIRO MAS (Eds.). Contribuições a Geologia da Amazônia, Manaus, 2: 201-224.

LUIZ JG \& SILVA LMC. 1995. Geofísica de prospecção. Universidade Federal do Pará. Belém, Editora Universitária, 311 p.

MACHADO N, LINDENMAYER Z, KROGH TE \& LINDENMAYER D. 1991. $\mathrm{U}-\mathrm{Pb}$ geochronology of Archean magmatism and basement reactivation in the Carajás area, Amazon shield, Brazil. Precambrian Research, 49: 329-354.

MADRUCCI V, VENEZIANI P \& PARADELLA WR. 2003. Caracterização Geológica e Estrutural através da Interpretação de Produto Integrado TMLandsat 5 e Dados Aerogamaespectrométricos, Região de Alta Floresta, MT. Revista Brasileira de Geofísica. 21(3): 219-234.

MINTY BRS. 1991. Simple Micro-Levelling for Aeromagnetic Data. Exploration Geophysics, 22: 591-592.

NOGUEIRA ACR, TRUCKENBRODT W \& PINHEIRO RVL. 1995. Formação Águas Claras, Pré-Cambriano da Serra dos Carajás. Redescrição e redefinição. Boletim do Museu Paraense Emílio Goeldi, Série Ciências da Terra, 7: 177-197.

PARADELLA W, BIGNELLI PA, VENEZIANI P, PIETSCH RW \& TOUTIN T. 1997. Airborne and spaceborne Synthetic Aperture Radar (SAR) integration with Landsat TM and gamma ray spectrometry for geological mapping in a tropical rainforest environment, the Carajás Mineral Province, Brazil. International Journal of Remote Sensing, 18: 1483-1501.

PARADELLA W, SANTOS AR dos, VENEZIANI P, SANT'ANNA MV \& MORAIS MC de. 2000. Geological investigation using RADARSAT-1 images in the tropical rain forest environment of Brazil. Canadian Journal of Remote Sensing, 26: 82-90.

PIDGEON RT, MACAMBIRA MJB \& LAFON JM. 2000. Th-U-Pb isotopic systems and internal structures of complex zircons from an enderbite from the Pium Complex, Carajás Province, Brazil: evidence for the ages of granulite facies metamorphism and the protolith of the enderbite. Chemical Geology, 166: 159-171.

NABIGHIAN MN, GRAUCH VJS, HANSEN RO, LaFEHR TR, LI Y, PEIRCE JW, PHILLIPS JD \& RUDER ME. 2005. The historical development of the magnetic method in exploration, Geophysics, 70: 33-61. 
PINHEIRO RVL. 1997. Reactivation history of the Carajás and Cinzento Strike-Slip Systems, Amazon, Brazil. Ph.D. Thesis, University of Durham, Durham, England, 408 p.

RABUS B, EINEDER M, ROTH A \& BAMLER R. 2003. The Shuttle Radar Topography Mission - a New Class of Elevation Models Acquired by Spaceborne Radar, Journal of Photogrammetry \& Remote Sensing, 57: 241-262.

RENCZ AN. 1999. Remote Sensing for the Earth Sciences. Manual of Remote Sensing, $3^{\text {rd }}$ edition, vol. 3. John Wiley/American Society for Photogrammetry and Remote Sensing. 707 pp.

SANTOS AR, PARADELLA WR, VENEZIANI P, LIU CC \& SANT'ANNA MV. 1997. Integração de dados SAR-TM e SAR-Gama em estudos geológicos na Província Mineral de Carajás (Brasil). In: VIII Simposio Latino-Americano de Percepción Remota/XVII Reunión Plenaria SELPER, Mérida. Caracas. CD-ROM.

SCHOWENGERDT RA. 1997. Remote sensing: models and methods for image processing. San Diego: Academic Press, 522 p.

SILVA AM. 1999. Integração de dados geológicos e geofísicos utilizandose uma nova técnica para seleção de alvos para exploração mineral, aplicada ao Greenstone Belt Rio das Velhas, Quadrilátero Ferrífero. Tese de Doutorado. Instituto de Geociências, Universidade de Brasília, 195 p.

SILVA GG, LIMA MJC, ANDRADE ARF, ISSLER RS \& GUIMARÃES G. 1974. Geologia das Folhas SB-22 Araguaia e parte SC-22 Tocantins, Projeto RADAM-BRASIL, geologia, geomorfologia, solos e uso potencial da terra, Levantamento de Recursos Naturais (4), Rio de Janeiro, 143 p.
TASSINARI CCG \& MACAMBIRA MJB. 2004. A evolução tectônica do Cráton Amazônico. In: XVII Congresso Brasileiro de Geologia, Araxá Recursos minerais e desenvolvimento socioeconômico: Anais... S.I.: SBG. CD-ROM.

TEIXEIRA AM. 2003. Integração de Dados Multifontes para Exploração de Ouro no Greenstone Belt Rio das Velhas, Quadrilátero Ferrífero, Minas Gerais. Instituto de Geociências, Universidade de Brasília, Brasília, Dissertação de Mestrado, 157 p.

TEIXEIRA AM, SILVA AM, PIRES ACB, MORAES RAV de \& SOUZA FILHO CR. 2004. Pesos de Evidência e sua Aplicação para Exploração Mineral em Greenstone Belts Arqueanos: 0 Exemplo do Supergrupo Rio das Velhas, Quadrilátero Ferrífero. In: I Simpósio Regional da Sociedade Brasileira de Geofísica. São Paulo. CD-ROM dos Anais do I Simpósio da Sociedade Brasileira de Geofísica.

TERUIYA RK. 2002. Integração Digital de Dados Multifontes no Estudo Geológico do Granito Cigano, Província Mineral de Carajás-PA. Dissertação de Mestrado, Instituto Nacional de Pesquisas Espaciais. $108 \mathrm{pp}$.

VENEZIANI P, SANTOS AR dos \& PARADELLA WR. 2004. A Evolução Tectono-estratigráfica da Província Mineral de Carajás: Um modelo com base em dados de Sensores Remotos orbitais (SAR-C Radarsat-1, TM Landsat-5), Aerogeofísica e Dados de Campo. Revista Brasileira de Geociências, 34(1): 67-78.

\section{NOTAS SOBRE OS AUTORES}

Cleyton de Carvalho Carneiro obteve o título de Técnico em Geomática com ênfase em Sensoriamento Remoto pelo Centro Federal de Educação Tecnológica do Pará - CEFET-PA (2002). Graduou-se em Geologia pela Universidade Federal do Pará - UFPA (2003). Titulou-se Mestre em Geociências na área de Metalogênese pela Universidade Estadual de Campinas - UNICAMP (2005). No ano de 2005 foi pesquisador do CNPq (DTI - categoria 7G). Atualmente desenvolve o doutorado em Geologia e Recursos Naturais no Instituto de Geociências da UNICAMP, junto ao Grupo de Geotecnologias. Atua no processamento e interpretação de imagens de Sensoriamento Remoto e Aerogeofísica, direcionado ao mapeamento geológico e prospecção mineral.

Alvaro Penteado Crósta graduou-se em Geologia pela Universidade de São Paulo (1977) e fez Mestrado e Doutorado em Sensoriamento Remoto, respectivamente pelo Instituto Nacional de Pesquisas Espaciais - INPE (1982) e Imperial College, Londres (1990). Realizou pós-doutorado junto ao Desert Research Institute, Universidade de Nevada, Reno, em 1995. É Professor Titular do Instituto de Geociências da Unicamp, onde atualmente exerce a função de Diretor. Atua em sensoriamento remoto e processamento digital de imagens, com ênfase em aplicações geológicas.

Adalene Moreira Silva é Engenheira Geóloga (1989) graduada pela Universidade Federal de Ouro Preto, Mestre (1992) e Doutora em Geologia (1999) pela Universidade de Brasília. Profissionalmente foi pesquisadora do Serviço Geológico Americano (1997-1999), Professora Visitante da Universidade de Brasília (2000-2002), Pesquisadora (05/2002-04/2003) e Professora Instituto de Geociências da UNICAMP (04/2003-10/2005). Atualmente é Professora do Instituto de Geociências da UnB. Professora Adalene vem ensinando e pesquisando sobre novas técnicas de processamento, interpretação e integração de dados aplicados a exploração mineral, hidrogeologia e ambiente. Entre 1997 e 2003 recebeu auxílios à pesquisa do CNPq, CAPES, FAPESP, USGS (EUA), Programa Petrobras Ambiental.

Roberto Vizeu Lima Pinheiro graduou-se em Geologia pela Universidade Federal do Pará em 1983, obtendo em 1986 o título de Mestre em Geologia pelo CPGGUFPA e de Doutor em Geologia Estrutural pela University of Durham (Inglaterra) em 1997. É Professor Adjunto do Departamento de Geologia da UFPA e professor do Curso de Pós-Graduação em Geologia e Geoquímica UFPA, atuando na área de Geologia Estrutural e Evolução Crustal. 